\title{
Comparison learning algorithms for artificial neural network model for flood forecasting, Chiang Mai, Thailand
}

\author{
T. Chaipimonplin \\ Department of Geography, Faculty of Social Sciences, Chiang Mai University, Chiang Mai, Thailand \\ Email: tawee.c@.cmu.ac.th
}

\begin{abstract}
Artificial neural network (ANN) has been widely used for flood forecasting, however, different learning algorithms may lead to different model performance. Therefore, this study investigated 10 learning algorithms; GDX (Variable Learning Rate Gradient Descent), BFG (BFGS Quasi-Newton), CGP (PolakRibiere Conjugate Gradient), BR (Bayesian Regularization), LM (Levenberg-Marquardt), OSS (One Step Secant), CGB (Conjugate Gradient with Powell/Beale Restarts, CGF (Fletcher-Powell Conjugate Gradient), SCG (Scaled Conjugate Gradient) and RP (Resilient Backpropagation).
\end{abstract}

This study area is located in the Upper Ping River Catchment, Chiang Mai, Thailand. There are two experiments for evaluating the learning algorithms performance. The first experiment is forecasting water level at P.1 station using water level and outflow from dam (7 input variables including: 5 variables of 3 water level stations (P.67t, P.67t-6, P.75, P.75t-6 and P. $1_{t}$ ) and 2 variables of outflow from the upper dam $\left(\right.$ Flow $_{\mathrm{t}}$ and Flow $\left.\mathrm{t}_{\mathrm{t}-6}\right)$. In addition, the second experiment is forecasting water level at P.67 station with using $\mathrm{dBz}$ value of Doppler radar images of precipitation, water level of the upper station and outflow from the dam (16 input variables including: 12 variables from 6 pixels of $\mathrm{dBz}\left(\mathrm{Z} 11_{\mathrm{t}}, \mathrm{Z} 11_{\mathrm{t}-6}, \mathrm{Z} 12_{\mathrm{t}}, \mathrm{Z} 12_{\mathrm{t}-6}, \ldots, \mathrm{Z} 23_{\mathrm{t}-6}\right), 2$

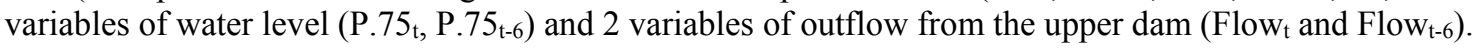

For ANN model development, model architecture was set with 1 hidden layer and a number of hidden nodes from 1 to $2 n+1$ nodes ( $n$ is the number of input variables) and forecasting times are 6,12 and 18 hours $(t+6$, $\mathrm{t}+12$ and $\mathrm{t}+18$ ). Available data for this study is only year 2005 with 5 flood events and the first flood event is for testing and other flood events are for training.

The results showed that increasing the number of hidden nodes did not affect the performance of the model excepted $\mathrm{t}+18$. Where, the performance of the model using LM and BR was decreased by increasing the number of hidden nodes. For the model performance with different learning algorithms, at P.1 station, all learning algorithms presented similar results in hydrographs at $t+6,12$ and 18 with CE: $0.02,0.03-0.05$, and 0.05-0.07 respectively. On the other hand, at P.67 station, all learning algorithms forecasted different hydrographs, BR and LM tended to be the best algorithm at $\mathrm{t}+6,12$ and 18 with CE: $0.37,0.33$ and $0.34 / 0.37$ respectively, while GDX was the worst algorithm with CE: $0.44,0.43$ and 0.49 respectively. Therefore, it could be said that the recommend algorithm for flood forecasting at P.1 and P.67 is LM algorithm because $\mathrm{LM}$ is a fast learning algorithm while BR is a slow learning.

Keywords: $\quad$ Artificial neural network, flood forecasting, learning algorithms, Thailand 


\section{INTRODUCTION}

Thailand is affected by flood almost every year, due to North-Eastern and South-Western monsoons, Chiang Mai, a big city in Thailand, suffers from floods as well. There are several studies that tried to forecast flooding in Thailand in order to reduce the effect of the flooding on the surrounded areas. Where, a related study to this article was carried out by Chaipimonplin et al. (2011a) using artificial neural network for flood forecasting at P.1 station and compared 2 learning algorithms; Levenberg-Marquardt (LM) and Bayesian Regularization (BR). The results of this study indicated that BR is better than LM, which is the same result for Chaipimonplin and Vangpaisal $(2014,2015)$ who compared LM and BR learning algorithms for flood forecasting at M.7, Mun Basin and pointed out that both learning algorithms predicted similar results but at the peak level of flood event, BR forecasted better than LM. In contrast, Chaipimonplin (2016) found LM is better than BR. In addition, Chaipimonplin et al. (2011b) tried to use radar image as the input variable for artificial neural network model to forecast flooding at P.1 station, and their result showed that using radar image with water level data can increase the forecasting time and improve the model performance for flood forecasting at P.1 station.

There are several learning algorithms that are available in the MATLAB but most of them are based on the method of gradient or Jacobian (Bealt et al., 2017). Fun and Hagan (1996) compared three learning algorithms (Levenberg-Marquardt-LM, Steepest Ascent-SA and Resilient Backpropagation-Rprop) and concluded that LM was the best performance for many cases while Rprop and SA algorithms failed to converge or Beale et al (2011) investigated 9 learning algorithms with 6 different of datasets and they concluded that each learning algorithm type has different potential of solving different problems such as LM has performance better than other algorithm as result with low mean square error, it is also the fastest learning algorithm or RP is the fastest algorithm for pattern recognition problem or SCG tends to be a good choice for a wide variety of problems as SCG seems to be fast like LM or RP.

Therefore, different of learning algorithms have different performance, as Chaipimonplin (2016) compared 2 learning algorithms between LM and BR for flood forecasting at P.1 and P. 67 and he found that LM was better than BR for forecasting the peak stage. Therefore, this article is about the exploration the model performance with more learning algorithms for flood forecasting at P.1 and P.67, also the outflow from dam is included as the extra input variable for flood forecasting at P.67.

\section{STUDY AREA AND DATA}

P.1 and P.67 water level stations are located in The Upper Ping Catchment with approximately $23,000 \mathrm{~km}^{2}$. As shown in Fig 1, there are 6 water level stations and Mae Ngat SomboonChon Dam is located above P.75 station. This article aims to forecast flood at P.1 station because it is located in the city of Chiang Mai and the P. 67 station because the currently flood warning for P.1 station is based on the water level at P.67. Where, when the water level at P.67 reach 4.10 meter, it takes approximately 6-7 hr to flow from P.67 to P.1 station with water level 3.7 meter (this is the P.1 station's riverbank level). Therefore, if the model can forecast flood at P.67, as a result it may increase the flood warning time at P.1 station.

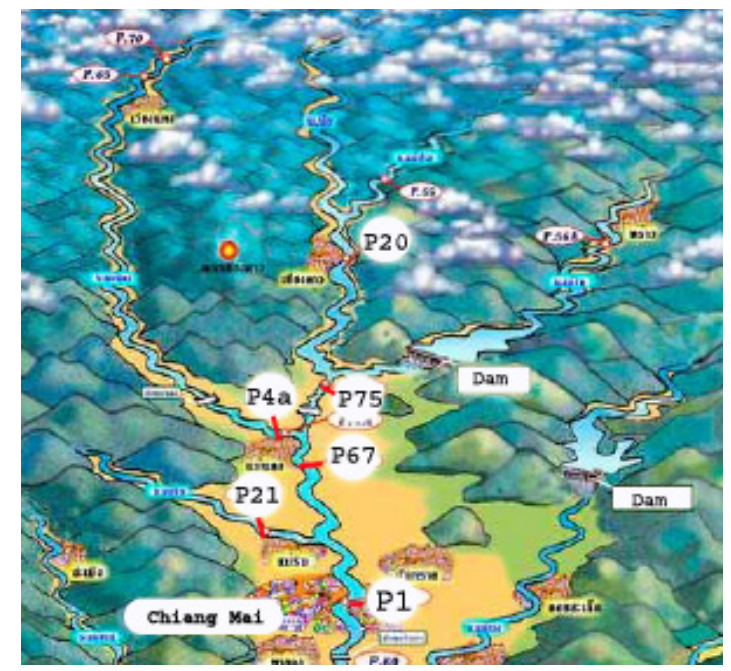

Figure 1. Study area and flood warning at P.1 station

Source: Hydrology and Water Management Centre for Upper Northern Region, (2007) 
However, in year 2005 most of rain gauge stations in Thailand record in daily, in order to obtain the precipitation in hourly interval, the Doppler radar images are required. The Doppler radar station that cover this study area is located at Omkoi District, Chiang Mai Province (Fig 2). Doppler radar is the equipment for detect the intensity of precipitation in the cloud and high $\mathrm{dBZ}$ value represents the high precipitation intensity and the spatial resolution is $1 \mathrm{~km}$.

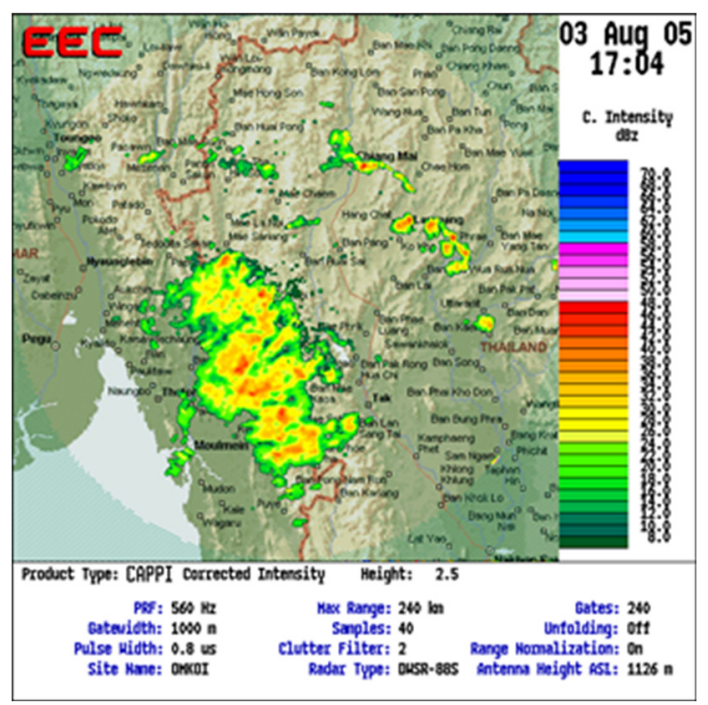

Figure 2. Doppler radar image

To extract the information of Doppler image from spatial information to point information (assume that each point represents as a rain gauge station), the sample points were set with 10 grids interval as the distance between each point is $10 \mathrm{~km}$. so for flood forecasting at P.67, was used only 6 pixels (Z11, Z12, Z13, Z21, Z22 and Z23) (Fig. 3) because it influences water level at P.67 station.

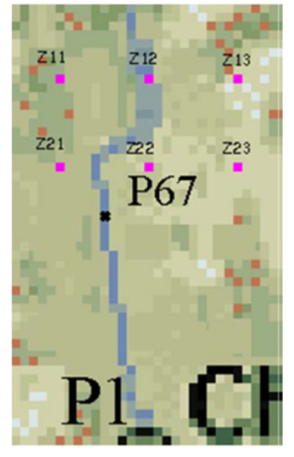

Figure 3. All 6 sample points

Data of this study are water level in hour at P.1, P.67 and P.75, the volume of water outflow from dam also the $\mathrm{dBz}$ value of the Doppler radar images. Due to the available data of Doppler image and flood event, so year 2005 are selected for this study. In 2005 between August and November, it was flood for 5 times with three big floods and two small floods (Fig. 4).

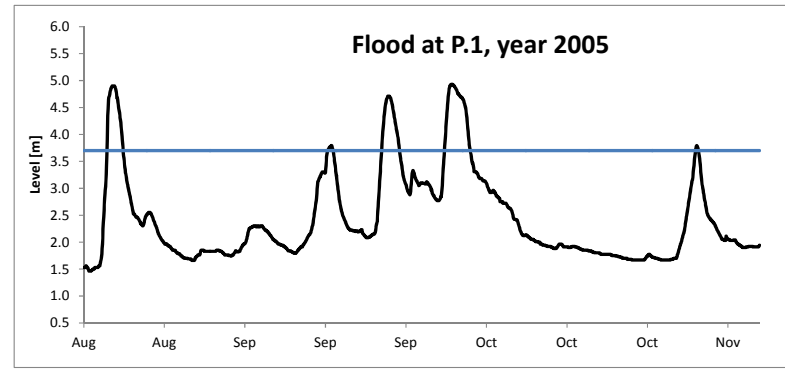

Figure 4. Hydrography at P.1 station 


\section{METHODOLOGY}

As the main objective is to investigate the performance of different algorithms, so input variables are fixed but only changing of learning algorithms and number of hidden nodes. All 10 learning algorithms that available in the Matlab software; Variable Learning Rate Gradient Descent (GDX), BFGS Quasi-Newton (BFG), Polak-Ribiere Conjugate Gradient (CGP), Bayesian Regularization (BR), Levenberg-Marquardt (LM), One Step Secant (OSS), Conjugate Gradient with Powell/Beale Restarts (CGB), Fletcher-Powell Conjugate Gradient (CGF), Scaled Conjugate Gradient (SCG) and Resilient Backpropagation (RP) and number of hidden nodes are increased from 1 node to $2 n+1$ nodes ( $n$ is the number of input variables) as Chaipimonlin (2010) reviewed lots of research papers and pointed out that the maximum number of suitable hidden nodes should be 2n+1 (Patuwo et al, 1993; Caudill, 1991)

There are 2 experiments of this study, one is forecasting water level at P.1 station with water level and out flow from dam (Flow) as the input variables and the second is forecasting water level at P.67 station with Doppler radar images and out flow from dam. For P.1 station input variables are water level at P. $1_{t}, P .67_{t}$, P.67t-6, P.75t, P.75t-6, Flow $\mathrm{t}_{\mathrm{t}}$ and Flow $\mathrm{t}_{\mathrm{t}-6}$ (7 input variables) and P.67 station are the $\mathrm{dBZ}$ value of 6 pixels at $\mathrm{t}$, $\mathrm{t}-6$, water level at P.75, P.75 $\mathrm{t}_{\mathrm{t}-6}$, Flow $\mathrm{t}$ and Flow $\mathrm{t}_{\mathrm{t}-6}$ (16 input variables). Due to the limitation of Doppler radar images with only 5 flood events available then for testing model performance all 4 events are for learning and 1 event is for testing (the first flood event) and the output of model is forecast at 6,12 and 18 hours in advance. For model performance evaluation the RMSE value is selected.

\section{RESULTS}

Fig 5 represents the error values of the models when increasing number of hidden nodes at P. 1 station. It is clearly that changing number of hidden nodes does not has any effects for model performance with different learning algorithms except learning algorithms BR and LM at $\mathrm{t}+18$ that the error values slightly increase when number of hidden node is increased. In addition, it is obviously that the RMSE at $\mathrm{t}+6$ of all model performances is less than 0.1 , while at $\mathrm{t}+12$ is approximately 0.2 .

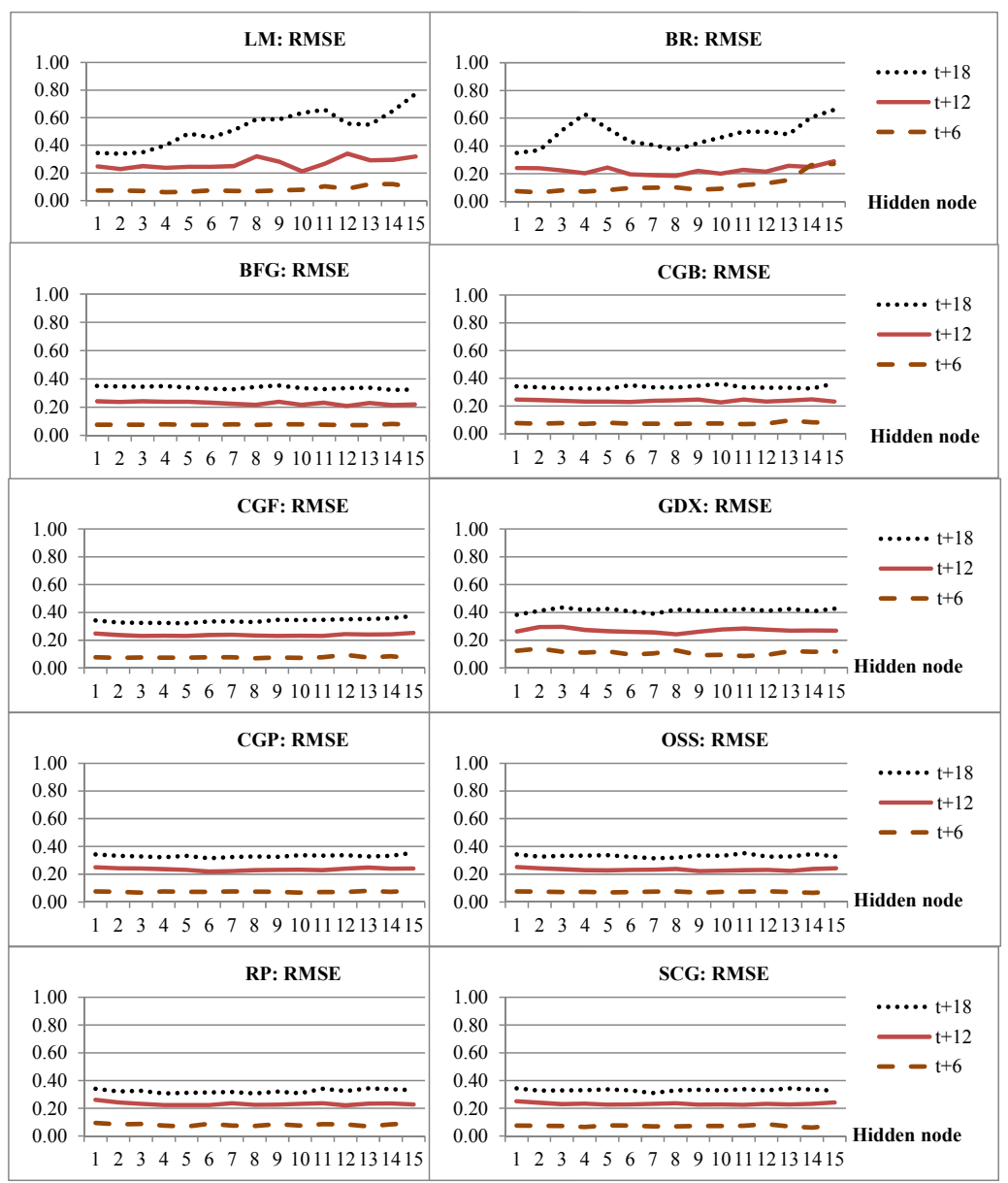

Figure 5. RMSE with hidden nodes at P.1 station 
Results at P.67 station are shown in Fig 6, it could be pointed out that it is two patterns of model performance when increasing number of hidden nodes; (1) RMSE value is increasing when number of hidden node is added up (BR and LM) and (2) when increasing number of hidden node, at the first rang of increasing hidden nodes (1 to 4 nodes), it improves model performance (RMSE values decrease), then adding more nodes, it has not much effect to the model performance (BFG, CGB, CGF, CGP, GDX, OSS, RP and SCG). In addition, the overall of model performance at $t+6,12$ and 18 is about 0.4 of RMSE.

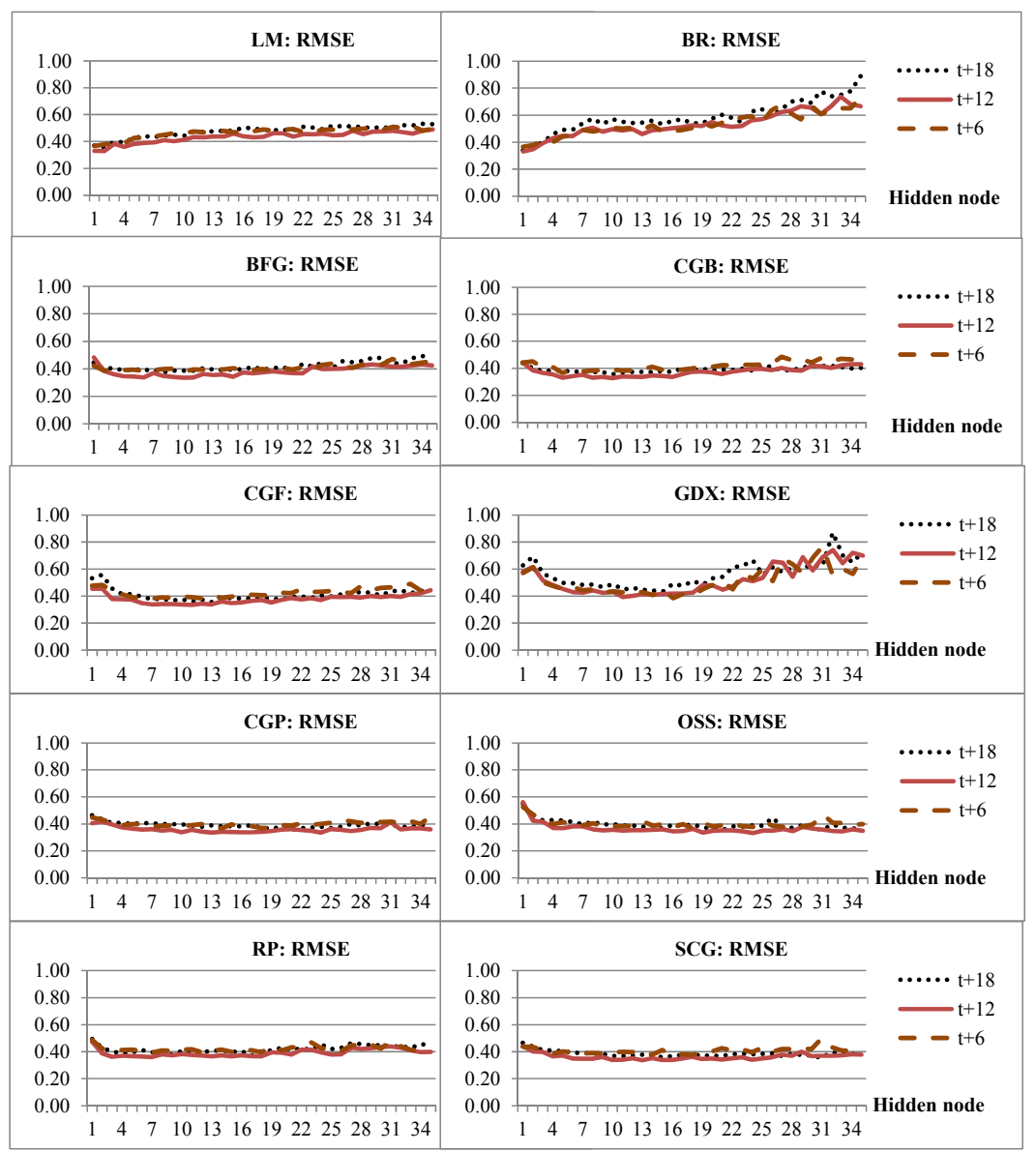

Figure 6. RMSE with hidden nodes at P.67 station

The models with the lowest RMSE value with different number of hidden nodes are selected to display in hydrography. (Fig 7). P.1 station with the core input variable is water level at $\mathrm{t}+6 \mathrm{hr}$, all models could forecast water level perfectly with slightly over forecasting at the peak stage but more error of forecasting the peak stage when increase leading time $(t+12$ and $t+18$ hr). For P.67 station with using radar image as the main input variables, artificial neural network model with different learning algorithms can forecast water level in different time steps from 6 up to $18 \mathrm{hr}$. Most of learning algorithms have the similar results for flood forecasting at P.1 and P.67 except GDX. 


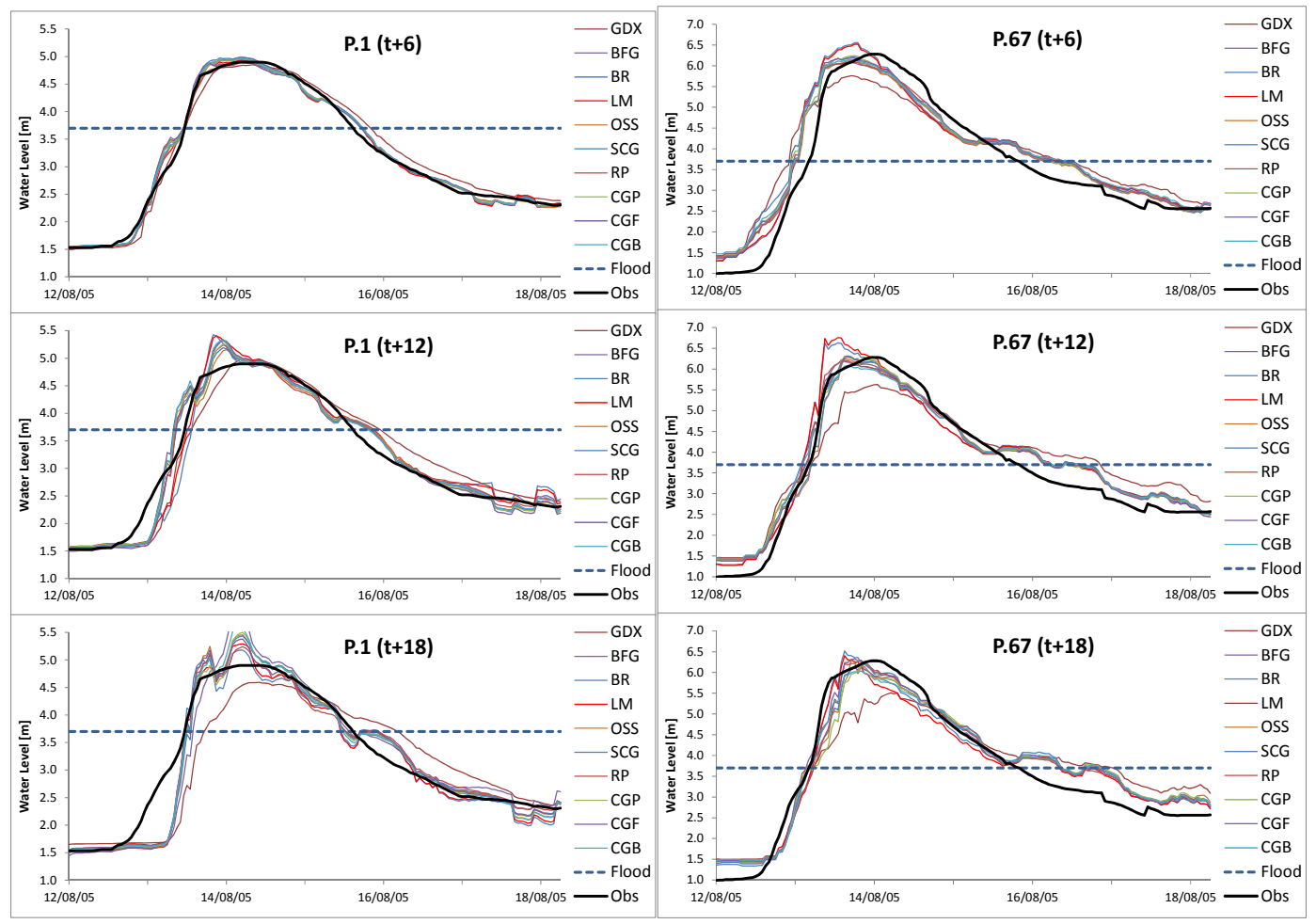

Figure 7. Hydrography of the best model performance at P.1 and P.67

\section{CONCLUSIONS}

In conclusion, most of learning algorithms forecasted similar results with both of P.1 and P.67 stations as input variables of each station are different types. For this study, flood forecasting at the Upper Ping Catchment, changing hidden nodes does not have influence model performance but only forecasting water level at $18 \mathrm{hr}$ in advances that the model performance is reduced while increasing hidden node in learning algorithms LM and BR. In addition, for the recommendation of the suitable learning algorithm for flood forecasting of both stations, LM would be recommended due to the speed of data processing. However, for other catchment might obtain different results.

\section{ACKNOWLEDGMENTS}

Chiang Mai University and Faculty of Social Sciences have kindly contributed financial support for this research. Thank you to Royal Irrigation Department for the water level data and data outflow from Mae Ngat Somboon Chon Dam., Department of Royal Rainmaking and Agricultural Aviation for radar images.

\section{REFERENCES}

Beale, M.H., M.T. Hagan, and H.B. Demuth (2011). Neural Network Toolbox ${ }^{T M 7}$ User's Guide, pp.951,. The Math Works, Inc. Natick.

Beale, M.H., M.T. Hagan, and H.B. Demuth. (2017). Neural Network Toolbox ${ }^{T M}$ User's Guide, pp 446, Retrieved June 5, 2017 from https://www.mathworks.com/help/pdf_doc/nnet/nnet_ug.pdf

Caudill, M. (1991). Neural network training tips and techniques. AI Expert, 6(1), 56-61.

Chaipimonplin, T. (2010). An Exploration of Neural Network Modelling Options for the Upper River Ping, Thailand. Ph.D. thesis, University of Leeds, Leeds.

Chaipimonplin, T. (2016). Investigation internal parameter of neural network model for flood forecasting at Upper River Ping, Chiang Mai. KSCE Journal of Civil Engineering, 20(1), 478-484.

Chaipimonplin, T., L. See, and P. Kneale. (2011a). Comparison of neural network learning algorithms: BR and LM for flood forecasting, Upper Ping Catchment. Poster presented at New Technologies for Urban Safety of Mega Cities in Asia (USMCA2011), Chiang Mai, Thailand, October 12-14. 
Chaipimonplin, T., L. See, and P. Kneale. (2011b). Improving neural network for flood forecasting using radar data on the Upper Ping River. Paper presented at $19^{\text {th }}$ International Congress on Modelling and Simulations (MODSIM2011), Perth, Australia, December 12-16.

Chaipimonplin, T., and T. Vangpaisal. (2015). The efficiency of input determination techniques in ANN for flood forecasting, Mun Basin, Thailand. Journal of Water Resource and Hydraulic Engineering, 4(2), 131-137.

Chaipimonplin, T., and T. Vangpaisal. (2015). The efficiency of input determination techniques in ANN for flood forecasting, Mun Basin, Thailand. International Journal of Computer and Electrical Engineering, $6(2), 90-94$.

Fun, M.H., and M.T. Hagan. (1996). Levenberg-Marquardt training for modular networks. Paper presented at the 1996 International Conference on Neural Networks, Washing ton, USA, June 3-6.

Hydrology and Water Management Centre for Upper Northern Region, (2007). Upper Northern Region Flood Warning Brochures, Retrieved November 16, 2007 from http://www.hydro-1.net/

Patuwo, E., M.Y. Hu, and M.S. Hung. (1993). Two group classification using neural network. Decision Sciences, 24(4), 825-845. 